\title{
Análisis metalexicográfico del Vocabulario de peruanismos de Miguel Ángel Ugarte Chamorro (Lima, 1997)
}

\author{
Luisa Portilla Durand \\ Departamento de Lingüística
}

$\mathrm{E}^{\prime}$

1 presente estudio pretende demostrar que, a pesar del tiempo transcurrido, el Vocabulario de Peruanismos del destacado investigador sanmarquino Miguel Ángel Ugarte Chamorro sigue constituyendo un hito en el quehacer lexicográfico de nuestro país y de San Marcos en particular.

Miguel Ángel Ugarte Chamorro (1910-1996) sintió, desde temprana edad, una curiosidad, siempre renovada, por las cosas del lenguaje. En un periodo que rebasó el medio siglo demostró un interés indesmayable por la caza de nuevas formas, modalidades y significados en el inagotable yacimiento lingüístico del castellano peruano. Hasta la última etapa de su existencia estuvo poseído por un vivo entusiasmo que lo llevó a coronar con éxito la obra de toda su vida: $E l$ vocabulario de peruanismos, trabajo de 311 páginas en el que se estiman 7300 entradas.

Hasta la última etapa de su vida, ya anciano y sufriendo de ceguera casi total, el doctor Miguel Ángel Ugarte no dejó de revisar pacientemente cada uno de los millares de peruanismos contenidos en este Vocabulario.

Su obra fue encargada al Fondo Editorial de la Universidad Nacional Mayor de San Marcos poco tiempo antes de su fallecimiento. La edición estaba ya en prensa al tiempo de su desaparición, así que de ella se hizo cargo su hermano, el destacadísimo hombre de teatro Guillermo Ugarte Chamorro.

\section{Macroestructura}

El Vocabulario expone, en su primera parte, una Advertencia, la Nota del editor, y la Presentación del autor y su obra. A continuación, se muestra el conjunto de 
entradas (más de 7000 ) y, para terminar, se presentan las Abreviaturas utilizadas y la Bibliografía.

Miguel Ángel Ugarte es el primer diccionarista del medio que replantea el viejo problema práctico de la ordenación alfabética de un inventario léxico. En esta línea, llama la atención en el Vocabulario la adopción del orden latino universal — vigente hace siglos en los grandes diccionarios europeos - que prescinde de los dígrafos $C h$ y $L l$ y los incluye dentro de los apartados correspondientes a las letras $C$ y $L$.

Las entradas que conforman la macroestructura del Vocabulario se escriben en letra negrita y minúscula (incluso la sigla $A P R A$, o nombres propios como Amazonía, Tahuantinsuyo, Tangüis aparecen en minúsculas). Sin embargo, voces como Collao «meseta del Collao», Cuba libre, o San Gil conservan la mayúscula inicial. (Llama la atención que Leal «nombre popular que se da al perro» figure también con mayúsculas.)

El uso de las marcas no se cumple siempre a cabalidad a lo largo del nutrido catálogo, por lo que se constatan ciertos desajustes en numerosos artículos. (Precisamos aquí que la información citada en el presente trabajo es copia fiel del Vocabulario). Por otro lado, la tabla de abreviaturas no es exhaustiva; así, no figuran, por ejemplo, formas como: d. = diminutivo; der. = derivado; vulg. = vulgar; inf. = informal; indet. = indeterminado. Asimismo, la citada Tabla se sitúa al final del Vocabulario, ubicación que dificulta la consulta rápida.

El diseño de un catálogo de entradas exige que éste se estructure con criterios coherentes y estables, tanto en el tratamiento como en la presentación del significado de las entradas; pero este Vocabulario muestra algunas carencias al respecto.

Por otra parte, se observan, sobre todo en las cabeceras de los artículos, algunos descuidos tipográficos en entradas como: bajos, baño, cunda, cazuela, mechadera, quemarse, ronda, sierra, tacazo, taclla, etc.

Finalmente, examinando el Vocabulario en su conjunto y dejando al margen algunos problemas técnicos en el tratamiento de las entradas y definiciones, 
creemos que el trabajo de Ugarte Chamorro se constituye en un interesante aporte en el cual tendrán que basarse las nuevas generaciones de lexicógrafos para constituir el vocabulario del español del Perú.

\section{PRINCIPALES IDEAS DEL PRÓLOGO}

La idea de Ugarte fue extender a todo el territorio nacional la tarea de recoger las voces familiares y populares no incluidas en el español general.

El autor inició su investigación en Arequipa, importante ciudad sureña del país con notable raigambre española, aunque con innegable influencia de las lenguas vernaculares: el quechua y el aimara. Por estas razones, esta zona sureña es la región donde convergen los vocablos indígenas y las voces populares del Cuzco, de Puno, de Moquegua y Tacna.

A partir de 1948 prosiguió su investigación en la UNMSM, desde la cátedra de lexicografía peruana. Lima, como capital del país, es la ciudad donde afluyen constantemente provincianos de las numerosas y variadas regiones de todo el territorio nacional, con el explicable propósito de asentarse en esta localidad para mejorar su situación cultural y económica.

Por otro lado, Ugarte basó su trabajo en otros autores que se ocuparon sobre peruanismos, pero no por ello dejó de lado a otros investigadores que trataron simplemente sobre localismos (v. gr. las voces referentes al oriente peruano o región amazónica fueron seleccionadas de la obra de Enrique Tovar Vocabulario del oriente peruano «y corresponden a las principales especies de flora, fauna y, así como a las costumbres y usos propios de la región»).

Los extranjerismos que se encuentran en el vocabulario, en su mayor parte anglicismos, son los más comunes entre los países sudamericanos, términos que difícilmente podrían ser reemplazados por voces españolas.

\section{ConcePto DE PERUANismo}

Queda implícito. (Véase el punto 2.) 


\section{FINALIDAD}

Es descriptiva, pues no hay criterios de corrección.

\section{CRITERIO DE CORRECCIÓN}

Como ya se indicó en el punto 4, no hay criterios de corrección.

\section{DEFINICIÓN DE DIFERENCIALIDAD}

Se plantea implícitamente: las entradas que aquí se presentan forman parte del léxico del español del Perú, independientemente de que las mismas voces puedan ser usadas en otros territorios de habla hispana.

\section{Tipos DE MATERIALES}

No hubo, propiamente, una selección: se trabajó con todo lo que se tenía a la mano.

\section{FUENTE DE LOS MATERIALES}

Se trata de información lexicográfica, trabajada, fundamentalmente, por aficionados, más no por estudiosos de la lexicografía, excepto en el caso del DRAE, que se usa como fuente de cotejo de algunas acepciones.

\section{LeNGuAs ESPECIALES}

—Sólo se hace evidente la marca Arq. (Arquitectura), la cual se utiliza una sola vez.

rellena. //f. (Arq.) Morcilla.

-Asimismo se observa la marca gros. (grosería)

arrechada. f. (gros.) Impulso lascivo.

cachero, ra. m. y f. (gros.) Lujurioso. 


\section{FLORA Y FAUNA}

- Sí se incluye abundante información de esta naturaleza. Al respecto se destacan los datos obtenidos del Vocabulario del oriente peruano de Enrique Tovar (aunque no se cita en la Bibliografía).

coriscapi. (Orte.) (Del q. cori: oro y caspi: madera) m. árbol que proporciona una madera muy utilizada en carpintería.

cornuda. f. (Orte.) Clase de rana que presenta sobre los ojos unas excrecencias corniformes. El macho tiene sobre los lados del pecho una especie de escudos espinosos.

\section{GeNTILICIOS}

- Hay abundante presencia de estas formas.

arequipeño, ña. adj. Natural de Arequipa. ú. t. c. s. // Perteneciente o relativo a esta ciudad o al departamento. Ac.

huanuqueño, ña. adj. Natural de Huánuco. ú. t. c. s. // Lo perteneciente o relativo a esta ciudad y departamento.

\section{HiPOCORÍSTICOS}

No se observa presencia de estas formas.

\section{SigLAS}

Sólo se presenta un caso de este tipo.

apra. m. Sigla del partido político: Alianza Popular Revolucionaria Americana.

\section{DefiniCión (SINONÍMICA, SEMÁNTICA, ENCICLOPÉDICA)}

—En el Vocabulario están presentes los tres tipos de definición.

acolchonar. tr. Acolchar. Ac.

buscapleitos . m. Picapleitos

ejemplarizador, ra. adj. Que da ejemplo.

frentazo. $\mathrm{m}$. Golpe dado con la frente o recibido en ella.

pacay. (Del q. pakay: esconder) m. Árbol de los valles cálidos cuyo fruto tiene forma de vaina larga, como un estuche de corteza verdosa y dura. Al 
abrirse ésta, deja ver, en serie, sus semillas negras y brillantes envueltas en una membrana algodonosa, jugosa y muy dulce. // El fruto de este árbol, llamado también guaba. Ac.

pachamanca. (Del q. pacha: tierra, suelo y manka: olla, y del aim. mankha: comida) f. Comida que generalmente se ofrece como agasajo y que consiste en abrir un gran hueco en el suelo, el que se calienta al rojo con leña y piedras que se cubren con capas de tierra, entre las que se ponen carnes aderezadas de toda especie, papas, camotes, yucas, choclos, queso fresco, plátanos, habas, etc. Ac.// fig. Confusión, desorden.

\section{SinÓNIMOS}

No se presentan.

16. MARCAS DE VARIACIÓN (CRONOLÓGICA, GEOGRÁFICA, SOCIAL, ESTILÍSTICA)

-Marcas cronológicas

$\begin{array}{ll}\text { desus. } & \text { desusado } \\ \text { m. ant. Inca } & \text { modo antigua Inca }\end{array}$

—Marcas geográficas

$\begin{array}{ll}\text { Abanc. } & \text { Abancay } \\ \text { Amaz. } & \text { Amazonas } \\ \text { Amé. } & \text { América } \\ \text { Anc. } & \text { Ancash } \\ \text { Apur. } & \text { Apurímac } \\ \text { Areq. } & \text { Arequipa } \\ \text { Ayac. } & \text { Ayacucho } \\ \text { Bol. } & \text { Bolivia } \\ \text { Caj. } & \text { Cajamarca } \\ \text { Cerro. P. } & \text { Cerro de Pasco } \\ \text { Col. } & \text { Colombia } \\ \text { Cuz. } & \text { Cuzco } \\ \text { Chicl. } & \text { Chiclayo } \\ \text { Chil. } & \text { Chile } \\ \text { Ecuad. } & \text { Ecuador }\end{array}$




$\begin{array}{ll}\text { Huanc. } & \text { Huancavelica } \\ \text { Huancay. } & \text { Huancayo } \\ \text { Huánu. } & \text { Huánuco } \\ \text { Huar. } & \text { Huaraz } \\ \text { Ica } & \text { Ica } \\ \text { Iquit. } & \text { Iquitos } \\ \text { Jun. } & \text { Junín } \\ \text { La Lib. } & \text { La Libertad } \\ \text { Lamb. } & \text { Lambayeque } \\ \text { Lim. } & \text { Lima } \\ \text { Lor. } & \text { Loreto } \\ \text { M. de Dios } & \text { Madre de Dios } \\ \text { Moq. } & \text { Moquegua } \\ \text { Orte. Merid. } & \text { Oriente Meridional } \\ \text { Piu. } & \text { Piura } \\ \text { Pun. } & \text { Puno } \\ \text { S. Mart. } & \text { San Martín } \\ \text { Tac. } & \text { Tacna } \\ \text { Truj. } & \text { Trujillo } \\ \text { Tumb. } & \text { Tumbes } \\ & \end{array}$

—Nivel de uso

fam. familiar pop. popular

—Intención del hablante

despect. despectivo

fest. festivo

gros. grosería

17. MaRCAS SISTÉMICAS (GRAMATICALES)

— Las marcas se detallan a continuación.

adj. adjetivo

adv. afirm. de afirmación 


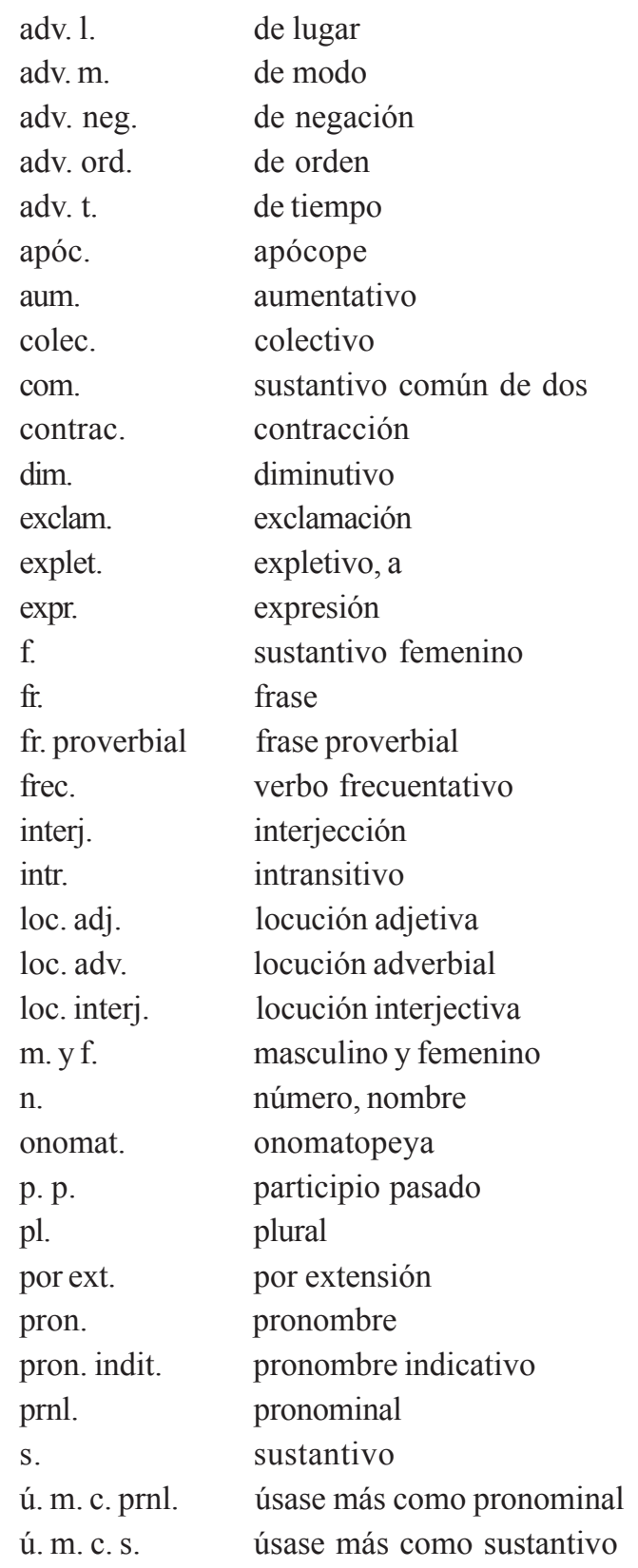


ú. m. en pl. úsase más en plural

ú. t. en sing. úsase también en singular

verb. verbo

18. Material PAREMiológico (REFRANES, MOdisMos, FRASES HeChas)

—A continuación se muestran algunos casos:

agua. $[. ..] / / ¡ A l$ agua patos! Frase que invita a proceder. [...]//Del agua mansa líbrenos Dios, que de la brava me libro yo. No hay que confiar en los que aparentan la humildad.

adivino. ¡Para los adivinos! Expresión que indica desconocer un hecho.

anchoveta. $[\ldots] / /$ Anchoveta no es pescado después de que te la has tragado. Censura contra los que son ingratos.

barriga. De los cuarenta para arriba no te mojes la barriga. Hay que cuidarse en la madurez. [...] // Barriga llena, corazón contento. Quien puede comer, queda satisfecho.

\section{EJEMPLIFICACIÓN}

- Se presenta sólo en ciertos casos:

cargar. // tr. fig. Enamorar a una mujer: ¿Por qué no le cargas a Juanita?

cascar. // (Del q. kachcay: roer) tr. Roer: Apúrate que te estoy cascando los talones.

\section{SISTEMAS DE REMISIÓN}

- Se observa dos tipos de remisión: endofórica (respecto al mismo vocabulario: se indica a través del subrayado) y exofórica en relación con el DRAE 84, 20. ${ }^{\text {a }}$ edición (para tal fin se usa la abreviatura Ac. 'Academia').

aluate. (Orte.) Nombre genérico que se da aciertos monos como la chuva, el cotomono, el frailecito, el pinchecillo, el macaquito o miquito y otros. El mono denominado guariba es más conocido con el nombre de aluate.

perezosa. f. Silla tumbona. Ac. 
21. Marcas, ABREVIATURAS y Símbolos

—Sólo se usan abreviaturas.

$\begin{array}{ll}\text { Abrev. } & \text { Abreviación } \\ \text { Ac. } & \text { Academia } \\ \text { aim. } & \text { aimara } \\ \text { afric. } & \text { africanismo } \\ \text { al. } & \text { alemán } \\ \text { Arq. } & \text { Arquitectura } \\ \text { Ecuat. } & \text { Ecuatoriano } \\ \text { fig. } & \text { figurado } \\ \text { fr. } & \text { francés } \\ \text { ingl. } & \text { inglés } \\ \text { it. } & \text { italiano } \\ \text { mejic. } & \text { mejicanismo } \\ \text { Neol. } & \text { Neologismo } \\ \text { Nor. } & \text { Norte } \\ \text { Orig. } & \text { origen } \\ \text { afric. } & \text { africanismo } \\ \text { Orte. } & \text { oriente } \\ \text { port. } & \text { portugués } \\ \text { posibl. } & \text { posible, probablemente } \\ \text { q. } & \text { quechua } \\ \text { Serr. } & \text { Serranía } \\ \text { Serr. Cent. } & \text { serranía central }\end{array}$

—Sólo se explicita un tipo de marca extralingüística:

// Otra acepción, otra frase

22. DoCUMENTACIÓN BIBLIOGRÁFICA

- La información no es completa. Las fuentes consignadas son las siguientes: 
Álvarez Vite, Juan

1989 Diccionario de peruanismos, Lima, Concytec.

Ángeles Caballero, César

1956 Los peruanismos en la literatura peruana, Lima.

Ayala L., Juan L.

1988 Diccionario Español-Aymara y Aymara-Español, Concytec, ediciones Mejía Baca.

Benvenuto Murrieta, Pedro

1932 Quince plazuelas y un callejón, Lima.

1936 El lenguaje peruano, Lima, Sanmarti y Cía.

CARRIÓN ORDÓÑEZ, Enrique

1978 "La formación del léxico español en la región andina" Sobretiro de la revista Lexis, Pontificia Universidad Católica del Perú.

1983 La Lengua en un texto de la ilustración, Lima Pontificia Universidad Católica del Perú.

Deza Galindo, Juan F.

1989 Diccionario Aymara-Castellano, Castellano-Aymara, Lima, Concytec,

Foley Gambetta, Enrique

1948 Léxico del Perú (8 fascículos).

Fray Domingo de Santo Tomás

1951 Lexicón o Vocabulario de la Lengua General del Perú. (Reproducción facsimilar.) Edición del Instituto de Historia de la Universidad de San Marcos.

GonzÁlez Holguín, Diego

1952 Vocabulario de la Lengua General de todo el Perú, llamada Lengua Quechua o del Inca, Lima, edición del Instituto de Historia de la Universidad de San Marcos.

Herrera, Fortunato L.

1939 Catálogo alfabético de los nombres vulgares y científicos de las plantas que existen en el Perú, Universidad de San Marcos.

HiLdebrandT Martha

1961 La lengua de Bolívar (Léxico), Universidad Central de Venezuela.

1969 Peruanismos, Lima, Francisco Moncloa Editores.

IBERICO MASS, Luis

1969 Cajamarquinismos, Cajamarca, Atahualpa. 
LIRA, Jorge

1944 Diccionario kkexhuwa-Español, Universidad de Tucumán.

Malaret, Augusto

1946 Diccionario de americanismos, Bs. As., Biblioteca Emecé.

Miró Quesada, Aurelio

1940 Costa, sierra y montaña, Lima.

NúÑEZ, Estuardo

1957 Suplemento al Diccionario de Peruanismos, Lima.

Real Academia Española

1984 Diccionario de la Lengua Española, Madrid, Espasa Calpe, S. A., 20. ${ }^{a}$ edición.

\section{TABÚ LINGÜísTICO}

—Sí se presenta. Para tal fin se usa la marca gros. (grosería)

culear. tr. (gros.) Realizar el acto sexual.

descartuchar. tr. (gros) Quitar la virginidad a una mujer. // prnl. Realizar el hombre el acto sexual por primera vez.

\section{INDIGENISMOS}

- Se presentan muchos casos, los cuales se reconocen por la información etimológica.

cherche. (Del q. chirche: niño llorón) adj. Criatura 1lorona.// Polluelo del gorrión.

choncholí. (Del q. chunchulli y del aim. ch'unch’uli: tripa) $\mathrm{m}$. Trocito de tripa de carnero que se fríe o asa a la parrilla. ú. m. en pl.

\section{EXTRANJERISMOS}

- Se reconocen gracias a la información etimológica.

bloc. (Del fr. bloc) m. Bloque.

chance. (Del ingl. chance) f. Ocasión, oportunidad, posibilidad. 


\section{Microestructura (MUestra y COMENTARios)}

-A lo largo del Vocabulario, se observan algunas irregularidades en la organización de la microestructura. Presentamos, a continuación, los artículos en los que se destaca la organización general de la microestructura del Vocabulario.

amarrarse.// prnl. fig. Casarse, contraer matrimonio. Ac. Amarrarse los pantalones: Fortalecer el Carácter ante una dificultad o peligro. // Amarrar el macho. Ociosear en el trabajo.

chic. adj. (del fr. chic) Elegante, que viste a la moda.// Gente chic: Gente distinguida.

espirituado, da. adj. (Piu.) Poseído por malos espíritus.

descascarar. // tr. Desconchar. ú. t. c. prnl. La pared se está descascarando.

—En los casos en que las palabras proceden de una lengua amerindia o extranjera, se presenta la marca etimológica inmediatamente después de la entrada. Seguidamente se ubica la marca gramatical y después la marca diatópica, de nivel de uso, de intención del hablante, según correspondan. Cuando se suma una acepción a las ya establecidas por el uso, se utiliza una doble barra diagonal. En los casos de remisión exofórica se escribe, detrás de la acepción, la abreviatura Ac. (Academia). Para indicar la etimología, así como para destacar las formas complejas, se utiliza el subrayado. (Lo mismo se hace en el caso de la remisión endofórica. Véase punto 20.) En el caso de presentarse ejemplos, éstos se muestran en letras cursivas.

\section{ANTECEDENTES DEL DiCCIONARIO}

El autor explicita sus deudas al Dr. Juan Corominas y a don Manuel Alvar, así como a quienes de manera indirecta permitieron la culminación del Vocabulario a través de su aporte bibliográfico.

\section{INFORMACIÓN ETIMOLÓGICA}

- Sí se presenta, sobre todo en entradas de procedencia quechua y aimara, y en el caso de los extranjerismos.

chonta. (Del q. chunta: madera de palmera muy dura) f. Clase de palmera de madera muy dura con la que se hacen arcos, flechas, macanas y bastones. El cogollo de esta palmera que se llama palmito es comestible. Ac. 
chupo. (Del q. chhupu y del aim. ch'upu: divieso) m. Forúnculo.

pichina. (Del it. piccino) f. Tubito de caucho por donde se inyecta aire a los balones deportivos.

\section{BiBLIOGRAFÍA}

AlCocer, Augusto

2000 "Reseña al Vocabulario de Peruanismos de Miguel Ángel Ugarte Chamorro" en la revista Letras de la Facultad de Letras de la UNMSM, Año 69, N. ${ }^{\circ}$ 95-96, pp. 271-5, Lima, UNMSM.

Martínez de Sousa, José

1995 Diccionario de lexicografía práctica, Barcelona, Bibliograf.

Portilla DuRand, Luisa P.

2002 "Vocabulario diferencial, contrastivo y ejemplificado del español del Perú", en la revista Escritura y Pensamiento de la Facultad de Letras de la UNMSM, año V, N. ${ }^{\circ}$ 9, pp. 139-149, Lima, UNMSM.

Real Academia Española

1984 Diccionario de la Lengua Española, Madrid, Espasa Calpe S. A., 20. ${ }^{\mathrm{a}}$ edición. 